\title{
A 3D deep learning approach to epicardial fat segmentation in non-contrast and post-contrast cardiac CT images
}

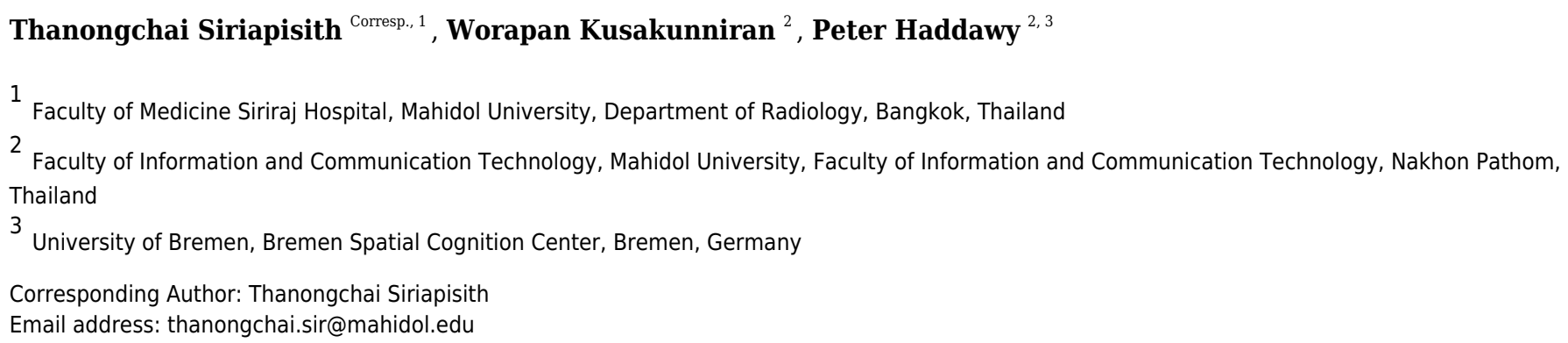

Epicardial fat (ECF) is localized fat surrounding the heart muscle or myocardium and enclosed by the thin-layer pericardium membrane. Segmenting the ECF is one of the most difficult medical image segmentation tasks. Since the epicardial fat is infiltrated into the groove between cardiac chambers and is contiguous with cardiac muscle, segmentation requires location and voxel intensity. Recently, deep learning methods have been effectively used to solve medical image segmentation problems in several domains with state-of-the-art performance. This paper presents a novel approach to 3D segmentation of ECF by integrating attention gates and deep supervision into the 3D U-Net deep learning architecture. The proposed method shows significant improvement of the segmentation performance, when compared with standard 3D U-Net. The experiments show excellent performance on non-contrast CT datasets with average Dice scores of $90.06 \%$. Transfer learning from a pre-trained model of a non-contrast CT to contrast-enhanced CT dataset was also performed. The segmentation accuracy on the contrast-enhanced CT dataset achieved a Dice score of $88.16 \%$. 


\section{Manuscript Title}

2

3 A 3D deep learning approach to epicardial fat segmentation in non-

4 contrast and post-contrast cardiac CT images

5

6

7

8

${ }^{1}$ Department of Radiology, Faculty of Medicine Siriraj Hospital, Mahidol University, Bangkok, 11 Thailand

$12{ }^{2}$ Faculty of Information and Communication Technology, Mahidol University, Nakhon Pathom, 13 Thailand

$14{ }^{3}$ Bremen Spatial Cognition Center, University of Bremen, Germany

15

16

17
Corresponding Author:

Thanongchai Siriapisith ${ }^{1}$

Arun-Amarin, Bangkoknoi, Bangkok, 10700, Thailand

Email address: thanongchai.sir@mahidol.edu

Keyword: epicardial fat, computed tomography, 3D segmentation, deep learning, 3D U-Net, attention gate, deep supervision 
40

41

42

43

44

45

46

47

48

49

50

51

52

53

54

55

56

57

58

59

60

61

62

63

64

65

66

67

68

69

70

71

72

73

74

75

76

77

78

79

\section{Abstract}

Epicardial fat (ECF) is localized fat surrounding the heart muscle or myocardium and enclosed by the thin-layer pericardium membrane. Segmenting the ECF is one of the most difficult medical image segmentation tasks. Since the epicardial fat is infiltrated into the groove between cardiac chambers and is contiguous with cardiac muscle, segmentation requires location and voxel intensity. Recently, deep learning methods have been effectively used to solve medical image segmentation problems in several domains with state-of-the-art performance. This paper presents a novel approach to 3D segmentation of ECF by integrating attention gates and deep supervision into the 3D U-Net deep learning architecture. The proposed method shows significant improvement of the segmentation performance, when compared with standard 3D UNet. The experiments show excellent performance on non-contrast CT datasets with average Dice scores of $90.06 \%$. Transfer learning from a pre-trained model of a non-contrast CT to contrast-enhanced CT dataset was also performed. The segmentation accuracy on the contrastenhanced CT dataset achieved a Dice score of $88.16 \%$.

\section{Introduction}

Epicardial fat (ECF) is localized fat surrounding the heart muscle and enclosed by and located inside the thin-layer pericardium membrane. The adipose tissue located outside the pericardium is called paracardial fat [1] and is contiguous with other mediastinal fat (Fig 1). ECF is the source of pro-inflammatory mediators and promotes the development of atherosclerosis of coronary arteries. The clinical significance of the ECF volume lies in its relation to major adverse cardiovascular events. Thus, measuring its volume is important in diagnosis and prognosis of cardiac conditions. ECF volume can be measured in non-contrast CT images (NCCT) with coronary calcium scoring and in contrast-enhanced CT images (CECT) with coronary CT angiography (CCTA). However, accurate measurement of ECF is challenging. The ECF is separated from other mediastinal fat by the thin pericardium. The pericardium is often not fully visible in CT images, which makes the detection of the boundaries of ECF difficult. ECF can also be infiltrated into grooves between cardiac chambers and is contiguous to the heart muscle. These technical challenges not only make accurate volume estimation difficult but make manual measurement a time-consuming process that is not practical in routine use. Therefore, computer-assisted tools are essential to reduce the processing time for ECF volume measurement.

Automated segmentation could potentially make ECF volume estimation more practical on a routine basis. Several approaches based on prior medical knowledge or non-deep learning techniques have been proposed for ECF segmentation, including genetic algorithms, region-ofinterest selection with thresholding, and fuzzy c-means clustering [2-4]. Deep learning techniques have been applied to a wide variety of medical image segmentation problems with great success [5-7]. A recent article [8] demonstrates that deep learning algorithms outperform conventional methods for medical image segmentation in terms of accuracy. But most previous 
80

81

82

83

84

85

86

87

88

89

90

91

92

93

94

95

96

97

98

99

100

101

102

103

104

105

106

107

108

109

110

111

112

113

114

115

116

117

118

119

studies involved large solid organs or tumor segmentation [9-11]. The segmentation of relatively small and complex structures with high inter-patient variability, such as ECF, has been far less successful. Recently, a few deep learning approaches to ECF segmentation have made progress on this problem [12-14]. In this paper, we build upon the previous work by presenting a novel deep learning model for 3D segmentation of ECF, We propose a solution of automatic segmentation of ECF volume using a deep learning based approach that is evaluated on both non-contrast and contrast-enhanced CT datasets. The NCCT dataset uses coronary calcium scoring and the CECT dataset uses contrast-enhanced coronary CT angiography (CE-CCTA). The model is first learned from scratch on the NCCT dataset with coronary calcium scoring CT. To cover the entire heart, it is scanned in 64 slices with $2.5 \mathrm{~mm}$ thickness on each acquisition. Then, the model pre-trained on that NCCT dataset is transferred to the CECT dataset which uses CE-CCTA. The CE-CCTA study is performed in 256 slices with $0.625 \mathrm{~mm}$ thickness

One of the key contributions of this paper is that we validate the performance of our newly developed 3D CNN-based approach on these difficult tasks. Since segmentation of ECF requires utilization of both voxel intensity and location information, we integrate two attention gate (AG) and deep supervision modules (DSV)into a standard 3D U-Net architecture. Our proposed model has better performance than the recent state-of-the-art approaches we evaluate because of the integration of AG and DSV modules. The AG module is used to focus on the target structures by suppressing irrelevant regions in the input image. The DSV module is used to increase the number of learned features by generating a secondary segmentation map combining the different resolution levels of network layers. The second main contribution is the use of transfer learning, taking a model pre-trained on NCCT data, and applying it to CECT data, using only a small amount of data for the re-training. This approach has benefits in clinical applications for both NCCT and CECT data for ECF segmentation. Furthermore, our proposed solution is 3D-based and does not require preprocessing and postprocessing steps, thus it can easily integrate into the clinical workflow of CT acquisition to rapidly generate ECF volume results for the physician in clinical practice.

\section{Related works}

Conventional non-deep learning methods have been proposed for ECF segmentation. Rodrigues et al. [2] proposed a genetic algorithm to recognize the pericardium contour on CT images. Militello et al. [3] proposed a semi-automatic approach using manual region-of-interest selection followed by thresholding segmentation. Zlokolica et al. [4] proposed local adaptive morphology and fuzzy c-means clustering. Rodrigues et al. [15] proposed ECF segmentation in CECT images using the Weka library (an open-source collection of machine learning algorithms) with Random-Forest as the classifier. The experiment, performed on 20 patients, yielded a Dice score of $97.7 \%$. However, these conventional methods required many preprocessing steps before entering the segmentation algorithm. The next evolution of ECF segmentations was performed with a deep learning approach. Commandeur et al. [14] proposed ECF segmentation from non- 
120

121

122

123

124

125

126

127

128

129

130

131

132

133

134

135

136

137

138

139

140

141

142

143

144

145

146

147

148

149

150

151

152

153

154

155

156

157

158

159

contrast coronary artery calcium computed tomography using ConvNets. They reported the Dice score of $82.3 \%$.

To improve the performance of medical image segmentation, several modifications of UNet [16] have been proposed. The spatial attention gate has been proposed to focus on the spatial and detailed structure of the important region varying in shape and size [17]. Schlemper et al. [17] demonstrate the performance of the attention U-Net on real-time fetal detection on $2 \mathrm{D}$ images and pancreas detection on 3D CT images. He et al. [12] proposed ECF segmentation from CE-CCTA using a modified 3D U-Net approach by adding attention gates (AG). AGs are commonly used in classification tasks [18-21] and have been applied for various medical image problems such as image classification [21, 22], image segmentation [17-22], and image captioning [22]. AG are used to focus on the relevant portion of the image by suppressing irrelevant regions [17]. The integration of $\mathrm{AG}$ into the standard U-Net [7, 11, 12, 17, 23] or VNet $[11,24]$ has been demonstrated to have benefits for region localization.

As mentioned above, the ECF has a complex structure. Some parts contain a thin layer adjacent to the cardiac muscle, which is similar to the microvasculature of the retinal vascular image visualized as small linear structures. In order to improve the performance of segmentation of small structures, several modules have been integrated into the main architecture of U-Net and V-Net, such as dense-layer and deep supervision modules [23-27]. The dense-layer [25, 26] has been used to enhance the segmentation result instead of the traditional convolution in the U-Net model. Deep supervision [23, 24, 27] was used to avoid local minimal traps during the training. The deep supervision helps to improve model convergence and increases the number of learned features [23]. Kearney et al. [23] showed that addition of deep supervision added to the U-Net model can improve the performance of 3D segmentation in CT images of the prostate gland, rectum, and penile bulb.

While 2D and 3D deep learning approaches have been used for medical image segmentation, 3D approaches have typically shown better performance than the 2D approaches $[9,10,28]$. For example, Zhou et al. [10] demonstrated the better performance of 3D CNN approaches on multiple organs on 3D CT images, when compared to the 2D based method. Starke et al. [9] also demonstrated that 3D CNN achieved better performance on segmentation of head and neck squamous cell carcinoma on CT images. Woo et al. [28] demonstrated that 3D UNet provided better performance on brain tissue MRI images, compared with 2D U-Net, on a smaller training dataset. Therefore, in this paper we use a 3D CNN for segmenting epicardial fat in cardiac $\mathrm{CT}$ images.

\section{Materials \& Methods}

\section{CNN architecture}

The model architecture is based on a 3D U-Net model composed of multiple levels of encoding and decoding paths. The initial number of features at the highest layers of the model is 32. The numbers of feature maps are doubled with each downsampling path. In addition to the 
160

161

162

163

164

165

166

167

168

169

170

171

172

173

174

175

176

177

178

179

180

181

182

183

184

185

186

187

188

189

190

191

192

193

194

195

196

197

198

199

original U-Net architecture, we added an attention gate connecting the encoding and decoding paths and deep supervision at the final step of the network. The model is created on a fully 3D structure at each network level. The final layer is an element-wise sum of feature maps of the two last decoding paths. The segmentation map of two classes (epicardial fat and background) is obtained using the output layer with threshold 0.5 to generate the binary classification of the epicardial fat. The architecture of the proposed network is shown in Fig 2.

Starting with the standard 3D U-Net architecture, the attention gate module connects each layer of encoding and decoding paths. The gating signal $(\mathrm{g})$ is chosen from the encoding path and the input features $(\mathrm{x})$ are collected from the decoding path. To generate the attention map, $\mathrm{g}$ and $\mathrm{x}$ go through a $1 \times 1 \times 1$ convolution layer and element-wise sum, followed by rectified linear unit (ReLu) activation, a channel-wise $1 \times 1 \times 1$ convolutional layer, batch normalization and a sigmoid activation layer. The output of sigmoid activation is concatenated to the input $\mathrm{x}$ to get the output of the attention gate module $[12,23]$.

Deep supervision $[11,24]$ is the module at the final step of the network where it generates the multiple segmentation maps at different resolution levels, which are then combined together. The secondary segmentation maps are created from each level of decoding paths which are then transposed by $1 \times 1 \times 1$ convolution. All feature maps are combined by element-wise sum. The lower resolution map is upsampled by $3 \mathrm{D}$ transposed convolution to have the same size as the second-lower resolution. Two maps are combined with element-wise sum then upsampled and added to the next level of segmentation map, until reaching the highest resolution level.

\section{CT imaging data}

This experimental study was approved and participant consent was waived by the institutional review board of Siriraj Hospital, Mahidol University (certificate of approval number Si 766/2020). The experimental datasets were acquired from 220 patients with non-contrast enhanced calcium scoring and 40 patients with CE-CCTA. The exclusion criteria were post open surgery of the chest wall. All CT acquisition was performed with the 256-slice multi-detector row CT scanner (Revolution CT; GE Medical Systems, Milwaukee, Wisconsin, United States). The original CT datasets of NCCT and CECT studies were 64 slices in $2.5 \mathrm{~mm}$ slice thickness and 256 slices in $0.625 \mathrm{~mm}$ slice thickness, respectively. All DICOM images were incorporated into a single 3D CT volume file with preserved original pixel intensity. Due to limitation of GPU memory, the 256 slices of CE-CCTA were pre-processed with rescaling to 64 images in the volume dataset. The final $3 \mathrm{D}$ volume dataset in all experiments was $512 \times 512 \times 64$. The dataset was raw 12 bits grayscale in each voxel. The area of pericardial fat was defined by fat tissue attenuation inside the pericardium, ranging from $-200 \mathrm{HU}$ to $-30 \mathrm{HU}[15,29,30]$. The groundtruth segmentation of ECF in all axial slices was performed using the 3D slicer software version 4.10.0 [31] by a cardiovascular radiologist with 18 years of experience. No additional feature map or augmentation was performed in the pre-processing step. 
200

201

202

203

204

205

206

207

208

209

210

211

212

213

214

215

216

217

218

219

220

221

222

223

224

225

226

227

228

229

230

231

232

233

234

235

236

237

238

239

\section{Training framework}

The experiments were implemented using the PyTorch (v1.8.0) deep learning library in Python (v3.6.9). The workflow for network training is illustrated in Fig 3. The training and testing processes were performed on a CUDA-enabled GPU (Nvidia DGX-A100) with 40 GB RAM. The experiments were divided into three scenarios: model validity assessment, NCCT, and CECT experiments. The parameters were the same for all three experiments. The networks were trained with RMSprop optimizer and mean squared error loss. The training parameters of learning rate, weight decay, and momentum were le-3, le- 8 and 0.9 , respectively. The initial random seed was set to be 0 . The illustration of the experimental framework is shown in Fig 3 .

The first experiment was the assessment of the model validity, for which we used 5-fold cross validation. The total dataset consisted of 200 volume-sets (12,800 images), divided into five independent folds. Each fold contained 160 volume-sets (10,240 images) for training and 40 volume-sets $(2,560$ images $)$ for validation, without repeated validation data between folds. The other 20 volume-sets (1,280 images) were left for testing in the second and third experiments. Then the 5-fold cross validation was performed on standard U-Net, AG-U-Net, DSV-U-Net, and the proposed method (AG-DSV-U-Net). For each fold of validation, the model with the best training accuracy after 150 epochs was selected for the validation.

The second experiment was to assess segmentation performance by training the network from scratch with the NCCT dataset. The volume matrix of each dataset was $512 \times 512 \times 64$ pixels. To compare the performance of segmentation, this experiment was performed with four model architectures: standard U-Net, AG-U-Net, DSV-U-Net, and the proposed method (AG-DSV-UNet). The network was evaluated with the hold-out method, in which a total of 220 volume-sets (14,080 images) were split into 200 volume-sets (12,800 images) for training (the same used in the first experiment) and 20 volume-sets (1,280 images) for testing. The model output was collected at the maximum number of iterations at the 300th epoch, named model-A.

The third experiment was to assess segmentation performance on the CECT dataset and to evaluate the effectiveness of transferring the learning from NCCT to the CECT datasets. The pre-trained 3D model (model-A) was trained on the large calcium scoring NCCT datasets. The key to the success of the transfer learning with 3D U-Net is to fine-tune only the shallow layers (contracting path) [32] instead of the whole network. This contracting path represents the more low-level features in the network [32]. The retraining dataset requires only a small amount of data - in our case only 20 volume-sets of CECT data. Note that these retraining cases are not from the same cases as used in the pre-trained model. To compare the performance of segmentation, this experiment was performed with four model architectures: standard U-Net, AG-U-Net, DSV-U-Net and proposed method (AG-DSV-U-Net). The network was evaluated with the hold-out method, in which the total 40 volume-sets (2,560 images) were split into 20 volume-sets (1,280 images) for training and 20 volume-sets (1,280 images) for testing. The output model is collected at the maximum number of iterations at of 300 thh epoch, named model-B. 
240

241

242

243

244

245

246

247

248

249

250

251

252

253

254

255

256

257

258

259

260

261

262

263

264

265

266

267

268

269

270

271

272

273

274

275

276

277

278

279

\section{Performance evaluation}

The performance of our proposed CNN segmentation is compared with the performance of the existing methods. The evaluation was quantitatively performed by comparison with the reference standard using the Dice similarity coefficient (DSC), Jaccard similarity coefficient (JSC), and Hausdorff distance (HD). An average HD value was calculated using the insight toolkit library of 3D slicer. Differences in the comparison coefficient among the four groups of experiments (standard U-Net, AG-U-Net, DSV-U-Net, and AG-DSV-U-Net) were assessed with a paired Student's t-test. $P$ values $<0.05$ indicated a statistically significant difference.

Differences in the comparison between DSC of segmentation result and ECF volume were assessed with Pearson's correlation coefficient. The Pearson's values of $<0.3$ indicated poor correlation, 0.3 to 0.7 indicated moderate correlation, and $>0.7$ indicated good correlation. To assess the consistency of the ground-truth, the inter-rater agreement was obtained by pixel-based correlation of DSC using two experienced cardiovascular radiologists with 18 and 14 years of experience. Ten volume sets (640 images) were randomly selected from NCCT dataset for the process of inter-rater correlation.

\section{Results}

The patient demographics are shown in Table 1. The training dataset consisting of 200 NCCT scan had an average age of 61.43 years and an average volume of $135.75 \mathrm{ml}$. The NCCT testing dataset had a similar distribution, with an average age of 67.80 years and an average volume of $127.59 \mathrm{ml}$. For the contrast-enhanced dataset, the average ages of the training and testing datasets were 65.85 and 60.85 years, respectively. The average volumes of ECF of the training and testing datasets were 117.13 and $121.43 \mathrm{ml}$, respectively. The inter-rater agreement of ECF ground-truth by two cardiovascular radiologists is about $91.73 . \pm 1.27 \%$, which indicates excellent correlation.

Five-fold cross validation experiments on our NCCT dataset were used to evaluate the validity and repeatability performance of the proposed method. The dataset was split into training (80\%) and validation (20\%) for each fold. On each model architecture, the validation data exhibits good results across each fold. The proposed method also demonstrates the best average performance ( $\mathrm{DSC}=89.02$ ), when compared with the other methods (at $\mathrm{p}<0.05$ when using validation data obtained in the five-fold cross-validation in the t-test), see Table 2.

The experimental result on the hold-out NCCT test dataset is shown in Table 3. The proposed method demonstrates excellent results, achieving average DSC, JSC, HD values of $90.06 \pm 4.60,82.42 \pm 6.91$ and $0.25 \pm 0.14$, respectively. The baseline of the experiment is the standard 3D U-Net, which demonstrates good results with DSC, JSC and HD values of $84.87 \pm 5.73,74.12 \pm 8.0 .8$ and $0.34 \pm 0.18$, respectively. The segmentation results of the modified U-Net models (AG-U-Net, DSV-U-Net, and the proposed method) demonstrate statistically significant improvement compared with the standard U-Net based on DSC $(p<0.05)$. The

Peer] Comput. Sci. reviewing PDF | (CS-2021:05:61572:2:0:NEW 8 Nov 2021) 
280

281

282

283

284

285

286

287

288

289

290

291

292

293

294

295

296

297

298

299

300

301

302

303

304

305

306

307

308

309

310

311

312

313

314

315

316

317

318

319

difference between segmentation results of AG-U-Net and DSV-U-Net is not statistically significant ( $\mathrm{p}>0.05$ ). The DSC, JSC, HD values of AG-U-Net are 89.59+4.45, 81.41+6.77 and $0.27+0.12$, respectively. The proposed method statistically improved the segmentation result $(p<0.05)$ compared with AG-U-Net as well. While the proposed method is better than DSV-UNet, the difference is not statistically significant $(\mathrm{p}>0.05)$. Examples of segmentation results of the proposed method are shown in Fig 4.

The experimental result of the proposed method on the CECT dataset is shown in Table 4. This transfer learning approach achieved average DSC, JSC and HD values of $88.16 \pm 4.57$, $79.10 \pm 6.75$ and $0.28 \pm 0.20$, respectively. The segmentation result of the proposed method demonstrates statistically significant improvement, when compared with the other methods based on DSC $(\mathrm{p}<0.0 .5)$. The segmentation results of the standard 3D U-Net and DSV-U-Net demonstrate good similar performance $(p>0.05)$. The segmentation results of the standard 3D UNet and DSV-U-Net are statistically significantly better than those of AG-U-Net $(p<0.05)$. Examples of segmentation results of transfer learning with the proposed methods are shown in Fig 5.

\section{Discussion}

Segmentation of ECF is a difficult image segmentation task because of the thin layer and complex structures at the outer surface of the heart. The ECF is also variable in distribution, depending on body habitus. In general, obese patients have larger amounts of ECF than do thin patients. Segmentation of ECF is more challenging than the segmentation of other cardiac structures.

Most CNN approaches work on 2D images whereas in clinical practice, 3D volume segmentation is used [33]. The 2D-based CNN approaches such as ResNet and VGG are not applicable for 3D datasets. The model architectures for 2D CNN and 3D CNN are different $[9$, 10,28]. 3D CNN has an advantage over 2D-CNN by extracting both spectral and spatial features simultaneously, while 2D CNN can extract only spatial features from the input data [5]. For this reason in general, the $3 \mathrm{D} \mathrm{CNN}$ is more accurate than a $2 \mathrm{D}$ one $[5,34] .2 .5 \mathrm{D} \mathrm{CNN}$ has been developed to solve the memory consumption problem of 3D models [35]. 2.5D CNN has at least three approaches $[35,36]$. The first is a combination of outputs of 2D CNNs in three orthogonal planes (axial, coronal, and sagittal) with majority voting. The second is to use 2D CNNs with 3 or 5 channels from adjacent 3 or 5 slices. Third is to apply 2D CNNs with randomly oriented $2 \mathrm{D}$ cross sections. In the final step, 2.5D segmentation requires an additional post-processing step to generate 3D output [37]. Although, the 3D CNN requires more resources and time for the model training, for the best performance, we use the 3D CNN in our implementation. The best performing methods for 3D volume segmentation of medical data are U-Net and V-Net. V-Net has more trainable parameters in its network architecture. Recent experimental comparisons of U-Net and V-Net on medical data have not shown statistically significant differences in performance $[12,38]$. However, U-Net is less complex and easier to 
320

321

322

323

324

325

326

327

328

329

330

331

332

333

334

335

336

337

338

339

340

341

342

343

344

345

346

347

348

349

350

351

352

353

354

355

356

357

358

359

modify so that additional modules can be integrated into the standard U-Net in order to improve the performance.

Several state-of-the-art approaches for CNN-based segmentation of ECF have recently been proposed. Commandeur et al. [14] proposed the first CNN-based method for ECF segmentation in a non-contrast 2D CT dataset using a multi-task convolutional neural network called ConvNets. They reported a Dice score of $82.3 \%$ for the segmentation result. He et al. [12] proposed another CNN-based method on a 3D CECT dataset using AG integrated into 3D UNet. The segmentation result was reported to have a DSC of $88.7 \%$ [12]. We repeated the experiment by implementing the AG in 3D U-Net on our NCCT dataset by hold-out testing. The amount of training data (200 volume-sets) is more than the one used in the previous article (150 volume-sets) [12]. Our 4 layer AG-U-Net method demonstrates significantly improved performance with DSC of $89.59 \%$, as compared with 3 layer AG-U-Net of $86.54 \%(\mathrm{p}<0.05)$. That should be due to more layers of our network. In our implementation, the AG-U-Net has more convolutional layers (4 layers) and removes the sigmoid at the end of the network. However, the AG integration provides significantly better performance $(\mathrm{p}<0.05)$ as compared with standard 3D U-Net (DSC of 84.87\%). To the best of our knowledge, our experiment uses

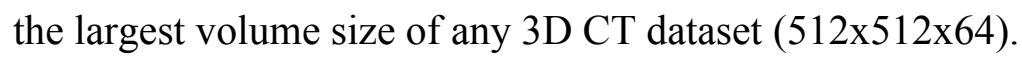

We introduce a novel approach to 3D segmentation of ECF by integrating both AG and DSV modules into all layers of the 3D U-Net deep learning architecture. The AGs are commonly used in natural image analysis and natural language processing [39, 40], which can generate attention-awareness features. The AG module is beneficial for organ localization, which can improve organ segmentation [17]. During CNN training, AG is automatically learned to focus on the target without additional supervision [41]. The AG module can improve model accuracy by suppressing feature activation in irrelevant regions of an input image [17]. The AG module is used to make connections between encoding and decoding paths on the standard U-Net. The DSV module is used to deal with the vanishing gradient problem in the deeper layers of a CNN $[11,27]$. The standard approach provides the supervision only at the output layer. But the DSV module propagates the supervision back to the earlier layer by generating a secondary segmentation map combining information from different resolution levels. The losses of this segmentation map are weighted and added to the final loss function and this can effectively increase the performance [42]. The DSV module is used by adding it into the decoding path of the 3D U-Net. The AG-DSV modules had been implemented in previous work [11,24] for kidney [24] tumor segmentation (Kidney Tumor Segmentation Challenge 2019), as well as for liver [11] and pancreas [11] tumor segmentation (Medical Decathlon Challenge 2018).

The experiment demonstrated that our proposed method (AG-DSV-U-Net) achieves excellent performance with average and max DSC values of $90.06 \%$ and $95.32 \%$, respectively. Our proposed method also shows a significant improvement of performance $(90.06 \%)$, when compared with the previous state-of-the-art network $(86.54 \%)$ on the same dataset $(\mathrm{p}<0.05)$. The example of the results was shown in Fig 4. While one might expect the segmentation performance to improve with fat volume of the dataset, unexpectedly, the statistical analysis 
360

361

362

363

364

365

366

367

368

369

370

371

372

373

374

375

376

377

378

379

380

381

382

383

384

385

386

387

388

389

390

391

392

393

394

395

396

397

398

399

demonstrates that there is poor correlation between segmentation performance and fat volume (Pearson's correlation 0.2).

The 3D volume size of our dataset is larger $(512 \times 512 \times 64)$ compared with the previous work $(512 \times 512 \times 32)$ [12]. For comparative analysis on different numbers of slices and image resolution of datasets, the previous work demonstrates that a 40-slice of volume dataset achieves $1 \%$ higher DSC than 32-slice and 24-slice [12]. However, the training time is also increased. We set up additional experiments to test the effect of different numbers of slices and image resolution on segmentation performance with our proposed model (AG-DSC-U-Net). The training, testing datasets, and hyperparameters are the same as defined in the NCCT experiment. The experiment of effect of the number of slices was performed by rescaling the slices with 64 , 32 and 16 slices and using a fixed image resolution with 512x512 pixels. The segmentation results are DSC $90.06 \%, 81.76 \%, 78.93 \%$, respectively. The experiment to determine the effect of different image resolution scales was performed by rescaling the resolution with $512 \times 512$, $256 \times 256$ and $128 \times 128$ pixels, with the number of slices fixed at 64 . The segmentation results are DSC $90.06 \%, 86.19 \%$ and $83.73 \%$ respectively. The $512 \times 512$ image resolution and 64 slices still give the best performance, with significant improvement over lower resolution $(\mathrm{p}<0.05)$. More slices and higher image resolution of the dataset let the network extract more spatial information that can help to improve segmentation accuracy. Furthermore, because the ECF is a thin layer along the heart contour, more spatial resolution will improve segmentation accuracy. To give the best performance, we chose 64 slices for our implementation which is a perfect fit with the original NCCT dataset, having 64 images in each dataset. In the CECT, the original CT dataset had 256 slices and needed to be rescaled to 64 slices. Due to the limitation of the proposed model and current GPU architecture, the voxel size of train and test datasets cannot be extended beyond 64 slices. The other limitation of this study is the size of the dataset: 220 volume-sets for NCCT experiment and 40 volume-sets for CECT experiment.

In clinical practice, the cardiac CT scan can be performed in NCCT or CECT or using both methods. For this reason, the ECF can also be either segmentation from NCCT or CECT dataset. To the best of our knowledge, ours is the first implementation of ECF segmentation on NCCT and CECT datasets. In our experiment, we started to train with the NCCT dataset (200 volume-sets). We used the concept of transfer learning to re-train with a similar dataset by taking a small amount of the dataset (Fig 3). We re-trained the pre-trained NCCT model with a small amount of CECT data (20 volume-sets). Weed test the model with additional testing of 20 volume-sets. The experimental result shows good performance with a DSC value of $88.16 \%$. The performance is also significantly better than that obtained with the standard U-Net and AG-UNet (Table 4). Training from scratch is more generally applicable but requires a large amount of training samples. Both NCCT and CECT datasets are similar in appearance but differ in color. The training from scratch approach of both datasets required a large amount of data samples. The transfer learning method is more practical because it uses smaller amounts of training data and still yields good performance. Additionally, our proposed re-trained model demonstrates good performance as compared with the previous training from scratch (88.7\%) [12]. 
400

401

402

403

404

405

406

407

408

409

410

411

412

413

414

415

416

417

418

419

420

421

422

423

424

425

426

427

428

429

430

431

432

433

434

435

436

437

438

439

440

441

Future studies could include investigations into more data diversity from multiple CT vendors, larger patient variation, and testing the model across different healthcare centers. Further investigation of clinical correlation between CNN segmentation of ECF volume and occurrence of cardiovascular disease is another interesting research questions.

\section{Conclusions}

We have introduced a CNN-based approach for ECF segmentation using integration of AG and DSV modules into the standard 3D U-Net. ECF segmentation is one of the most difficult medical image segmentation tasks. We trained the NCCT dataset from scratch and also re-trained on a CE CT dataset from the pre-trained NCCT model. We successfully improved the performance of ECF segmentation on both NCCT and CECT datasets when compared with the previous state-of-the-art methods. It is expected that this proposed method has the potential to improve the performance in other difficult segmentation tasks. The concept of training and retraining models can be also applied to other medical image segmentation problems.

\section{Acknowledgements}

Thank you to Dr.Jitladda Wasinrat who is the second cardiovascular radiologist for analysis of inter-rater agreement. This work was partially supported by a grant from the Mahidol University Office of International Relations to Haddawy in support of the Mahidol-Bremen Medical Informatics Research Unit.

\section{References}

[1]. Bertaso AG, Bertol D, Duncan BB, Foppa M. Epicardial fat: definition, measurements and systematic review of main outcomes. Arq Bras Cardiol. 2013;101(1):e18-e28.

[2]. Rodrigues ÉO, Rodrigues LO, Oliveira LSN, Conci A, Liatsis P. Automated recognition of the pericardium contour on processed CT images using genetic algorithms. Computers in Biology and Medicine. 2017;87:38-45.

[3]. Militello C, Rundo L, Toia P, Conti V, Russo G, Filorizzo C, Maffei E, Cademartiri F, La Grutta L, Midiri M, Vitabile S. A semi-automatic approach for epicardial adipose tissue segmentation and quantification on cardiac CT scans. Computers in Biology and Medicine. $2019 ; 114$.

[4]. Zlokolica V, Krstanović L, Velicki L, Popović B, Janev M, Obradović R, Ralević NM, Jovanov L, Babin D. Semiautomatic Epicardial Fat Segmentation Based on Fuzzy c-Means Clustering and Geometric Ellipse Fitting. Journal of Healthcare Engineering. 2017;2017. [5]. Singh SP, Wang L, Gupta S, Goli H, Padmanabhan P, Gulyás B. 3D Deep Learning on Medical Images: A Review. Sensors. 2020;20(18).

[6]. Kim M, Yun J, Cho Y, Shin K, Jang R, Bae H-J, Kim N. Deep Learning in Medical Imaging. Neurospine. 2019;16(4):657-68. 
442 [7]. Hesamian MH, Jia W, He X, Kennedy P. Deep Learning Techniques for Medical Image

443 Segmentation: Achievements and Challenges. Journal of Digital Imaging. 2019;32(4):582-96.

444 [8]. Renard F, Guedria S, Palma ND, Vuillerme N. Variability and reproducibility in deep

445 learning for medical image segmentation. Scientific Reports. 2020;10(1):13724.

446 [9]. Starke S, Leger S, Zwanenburg A, Leger K, Lohaus F, Linge A, Schreiber A, Kalinauskaite

447 G, Tinhofer I, Guberina N, Guberina M, Balermpas P, von der Grün J, Ganswindt U, Belka C,

448 Peeken JC, Combs SE, Boeke S, Zips D, Richter C, Troost EGC, Krause M, Baumann M, Löck

449 S. 2D and 3D convolutional neural networks for outcome modelling of locally advanced head

450 and neck squamous cell carcinoma. Sci Rep. 2020;10(1):15625.

451 [10]. Zhou X. Automatic Segmentation of Multiple Organs on 3D CT Images by Using Deep

452 Learning Approaches. In: Lee G, Fujita H, editors. Deep Learning in Medical Image Analysis :

453 Challenges and Applications. Cham: Springer International Publishing; 2020. p. 135-47.

454 [11]. Turečková A, Tureček T, Komínková Oplatková Z, Rodríguez-Sánchez A. Improving CT

455 Image Tumor Segmentation Through Deep Supervision and Attentional Gates. Frontiers in

456 Robotics and AI. 2020;7(106).

457 [12]. He X, Guo BJ, Lei Y, Wang T, Fu Y, Curran WJ, Zhang LJ, Liu T, Yang X. Automatic

458 segmentation and quantification of epicardial adipose tissue from coronary computed

459 tomography angiography. Physics in Medicine and Biology. 2020;65(9).

460 [13]. Rodrigues ÉO, Pinheiro VHA, Liatsis P, Conci A. Machine learning in the prediction of

461 cardiac epicardial and mediastinal fat volumes. Computers in Biology and Medicine.

462 2017;89:520-9.

463 [14]. Commandeur F, Goeller M, Betancur J, Cadet S, Doris M, Chen X, Berman DS, Slomka

464 PJ, Tamarappoo BK, Dey D. Deep Learning for Quantification of Epicardial and Thoracic

465 Adipose Tissue from Non-Contrast CT. IEEE Transactions on Medical Imaging.

466 2018;37(8):1835-46.

467 [15]. Rodrigues ÉO, Morais FFC, Morais NAOS, Conci LS, Neto LV, Conci A. A novel

468 approach for the automated segmentation and volume quantification of cardiac fats on computed

469 tomography. Computer Methods and Programs in Biomedicine. 2016;123:109-28.

470 [16]. Ronneberger O, Fischer P, Brox T, editors. U-Net: Convolutional Networks for

471 Biomedical Image Segmentation. Medical Image Computing and Computer-Assisted

472 Intervention - MICCAI 2015; 2015 2015//; Cham: Springer International Publishing.

473 [17]. Schlemper J, Oktay O, Schaap M, Heinrich M, Kainz B, Glocker B, Rueckert D. Attention

474 gated networks: Learning to leverage salient regions in medical images. Medical Image Analysis.

475 2019;53:197-207.

476 [18]. Wu Y, Zhang X, Xiao Y, Feng J. Attention Neural Network for Water Image

477 Classification under IoT Environment. Applied Sciences. 2020;10(3).

478 [19]. Sharmin S, Chakma D. Attention-based convolutional neural network for Bangla sentiment

479 analysis. AI \& SOCIETY. 2021;36(1):381-96.

480 [20]. Fei H, Zhang Y, Ren Y, Ji D. Optimizing Attention for Sequence Modeling via

481 Reinforcement Learning. IEEE Transactions on Neural Networks and Learning Systems. 2021:1-

48210.

483 [21]. Kelvin X, Jimmy B, Ryan K, Kyunghyun C, Aaron C, Ruslan S, Rich Z, Yoshua B. Show, 484 Attend and Tell: Neural Image Caption Generation with Visual Attention. 2015/06/01: PMLR; 485 2015. p. 2048-57. 
486 [22]. Zhao B, Feng J, Wu X, Yan S. A survey on deep learning-based fine-grained object 487 classification and semantic segmentation. International Journal of Automation and Computing. $488 \quad 2017 ; 14(2): 119-35$.

489 [23]. Kearney V, Chan JW, Wang T, Perry A, Yom SS, Solberg TD. Attention-enabled 3D 490 boosted convolutional neural networks for semantic CT segmentation using deep supervision. 491 Physics in Medicine \& Biology. 2019;64(13):135001.

492 [24]. Tureckova A, Turecek T, Komínková Z, Rodríguez-Sánchez A, editors. KiTS challenge: 493 VNet with attention gates and deep supervision2019.

494 [25]. Liang Z, Liu H, Zhao X, Yu L. Segmentation of Retinal Vessels Based on DenseNet495 Attention-Unet Model Network. Proceedings of the 2nd International Conference on Industrial 496 Control Network And System Engineering Research; Kuala Lumpur, Malaysia: Association for 497 Computing Machinery; 2020. p. 111-7.

498 [26]. Wu C, Zou Y, Zhan J. DA-U-Net: Densely Connected Convolutional Networks and 499 Decoder with Attention Gate for Retinal Vessel Segmentation. IOP Conference Series: Materials 500 Science and Engineering. 2019;533:012053.

501 [27]. Chen-Yu L, Saining X, Patrick G, Zhengyou Z, Zhuowen T. Deeply-Supervised Nets. 502 2015/02/21: PMLR; 2015. p. 562-70.

503 [28]. Woo B, Lee M, editors. Comparison of tissue segmentation performance between 2D U504 Net and 3D U-Net on brain MR Images. 2021 International Conference on Electronics, 505 Information, and Communication (ICEIC); 202131 Jan.-3 Feb. 2021.

506 [29]. Shahzad R, Bos D, Metz C, Rossi A, Kirişli H, van der Lugt A, Klein S, Witteman J, de 507 Feyter P, Niessen W, van Vliet L, van Walsum T. Automatic quantification of epicardial fat 508 volume on non-enhanced cardiac CT scans using a multi-atlas segmentation approach. Medical 509 Physics. 2013;40(9):091910.

510 [30]. Kazemi A, Keshtkar A, Rashidi S, Aslanabadi N, Khodadad B, Esmaeili M, editors. 511 Automated Segmentation of Cardiac Fats Based on Extraction of Textural Features from Non512 Contrast CT Images. 2020 25th International Computer Conference, Computer Society of Iran, 513 CSICC 2020; 2020.

514 [31]. Fedorov A, Beichel R, Kalpathy-Cramer J, Finet J, Fillion-Robin JC, Pujol S, Bauer C, 515 Jennings D, Fennessy F, Sonka M, Buatti J, Aylward S, Miller JV, Pieper S, Kikinis R. 3D Slicer 516 as an image computing platform for the Quantitative Imaging Network. Magn Reson Imaging. 517 2012;30(9):1323-41.

518 [32]. Amiri M, Brooks R, Rivaz H. Fine-Tuning U-Net for Ultrasound Image Segmentation: 519 Different Layers, Different Outcomes. IEEE Transactions on Ultrasonics, Ferroelectrics, and 520 Frequency Control. 2020;67(12):2510-8.

521 [33]. Milletari F, Navab N, Ahmadi S, editors. V-Net: Fully Convolutional Neural Networks for 522 Volumetric Medical Image Segmentation. 2016 Fourth International Conference on 3D Vision 523 (3DV); $201625-28$ Oct. 2016.

524 [34]. Han L, Chen Y, Li J, Zhong B, Lei Y, Sun M. Liver segmentation with 2.5D perpendicular 525 UNets. Computers \& Electrical Engineering. 2021;91:107118.

526 [35]. Zhang C, Hua Q, Chu Y, Wang P. Liver tumor segmentation using 2.5D UV-Net with 527 multi-scale convolution. Computers in Biology and Medicine. 2021;133:104424.

528 [36]. Minnema J, Wolff J, Koivisto J, Lucka F, Batenburg KJ, Forouzanfar T, van Eijnatten M. 529 Comparison of convolutional neural network training strategies for cone-beam CT image 530 segmentation. Computer Methods and Programs in Biomedicine. 2021;207:106192. 
531 [37]. Han Y, Li X, Wang B, Wang L. Boundary Loss-Based 2.5D Fully Convolutional Neural 532 Networks Approach for Segmentation: A Case Study of the Liver and Tumor on Computed 533 Tomography. Algorithms. 2021;14(5).

534 [38]. Ghavami N, Hu Y, Gibson E, Bonmati E, Emberton M, Moore CM, Barratt DC. Automatic 535 segmentation of prostate MRI using convolutional neural networks: Investigating the impact of 536 network architecture on the accuracy of volume measurement and MRI-ultrasound registration. 537 Medical Image Analysis. 2019;58:101558.

538 [39]. Wang F, Jiang M, Qian C, Yang S, Li C, Zhang H, Wang X, Tang X, editors. Residual 539 Attention Network for Image Classification. 2017 IEEE Conference on Computer Vision and 540 Pattern Recognition (CVPR); 2017 21-26 July 2017.

541 [40]. Anderson P, He X, Buehler C, Teney D, Johnson M, Gould S, Zhang L. Bottom-Up and 542 Top-Down Attention for Image Captioning and VQA. ArXiv. 2017;abs/1707.07998.

543 [41]. Oktay O, Schlemper J, Folgoc LL, Lee MJ, Heinrich M, Misawa K, Mori K, McDonagh 544 SG, Hammerla N, Kainz B, Glocker B, Rueckert D. Attention U-Net: Learning Where to Look 545 for the Pancreas. ArXiv. 2018;abs/1804.03999.

546 [42]. Kayalibay B, Jensen G, Smagt PVD. CNN-based Segmentation of Medical Imaging Data. 547 ArXiv. 2017;abs/1701.03056. 


\section{Figure 1}

The example CT dataset of epicardial fat

(A) is a non-contrast (B) is a post-contrast CT image. The pericardium is a thin layer of membrane surrounding the heart (arrow). The epicardial fat is fat along the outer surface of heart and inside the pericardium $(*)$.
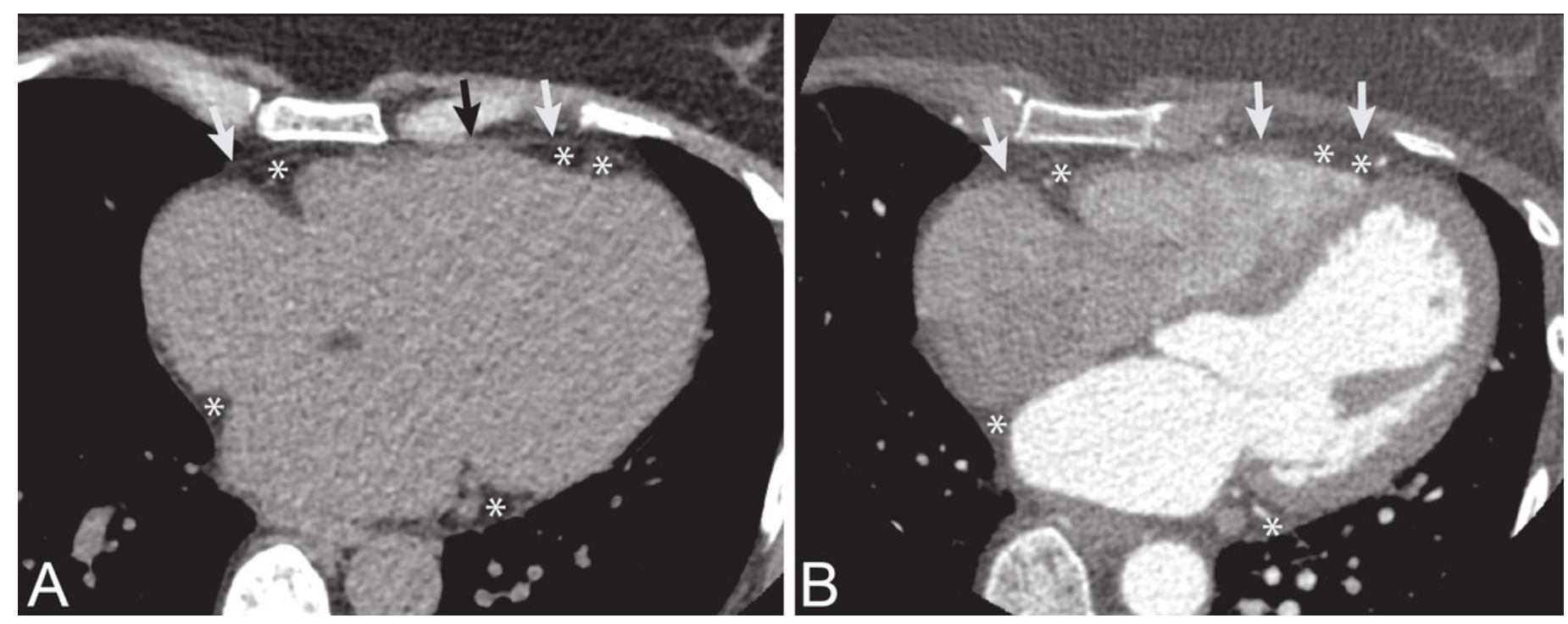
Figure 2

The proposed network for epicardial fat segmentation.

The network contains two main parts of the standard 3D U-Net integrated with the attention gate, and the deep supervision modules.

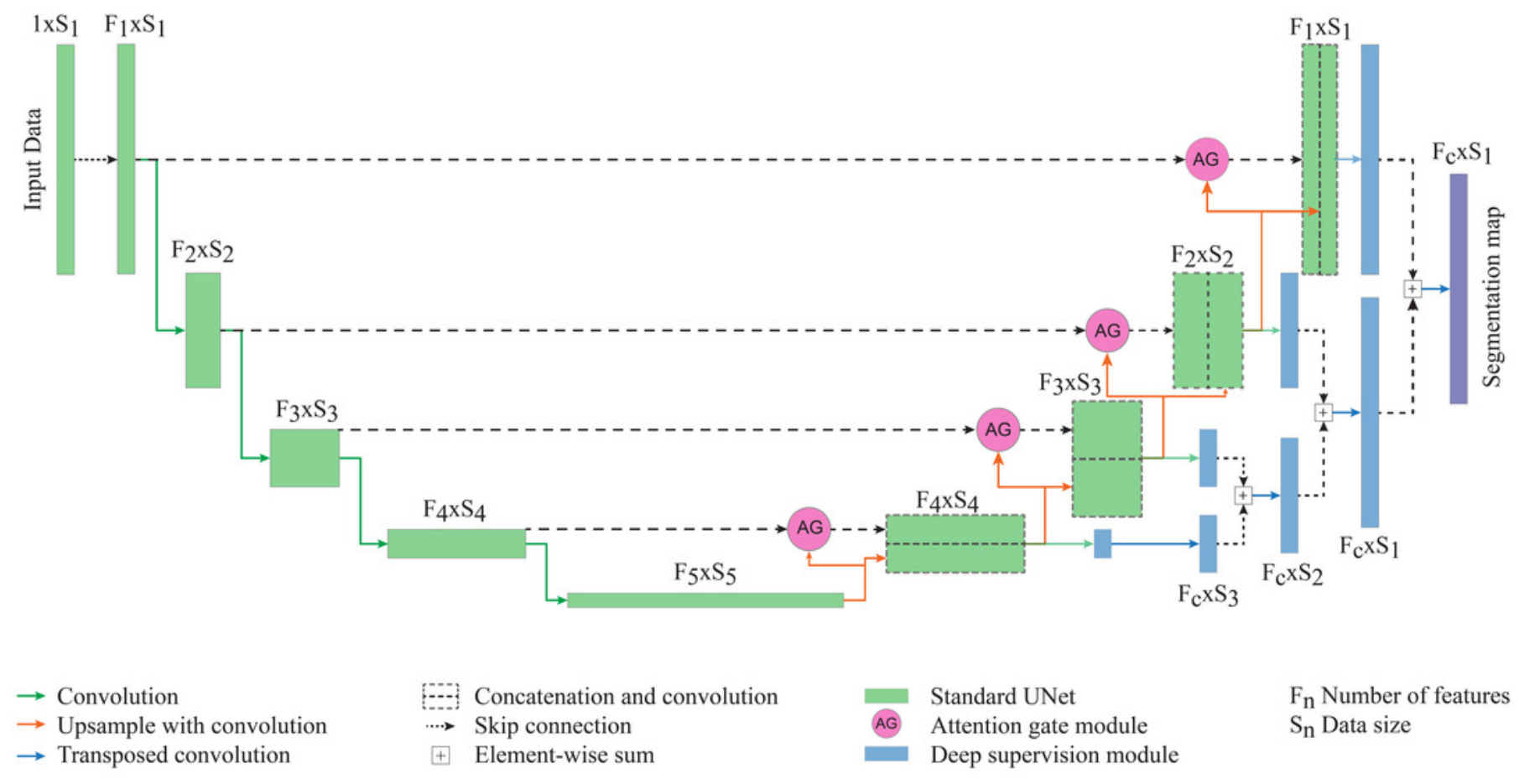




\section{Figure 3}

Illustration of framework of the proposed method.

The upper row performs the network training from scratch with a non-contrast CT dataset. The lower row performs the network re-training on a contrast-enhanced CT dataset. No postprocessing is required in this framework.

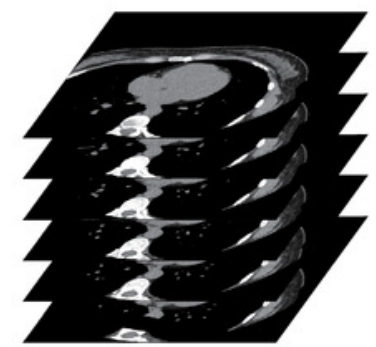

Non-contrast CT images

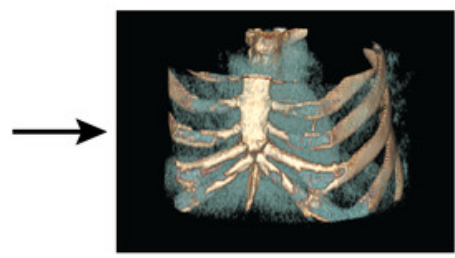

\section{D CT dataset}

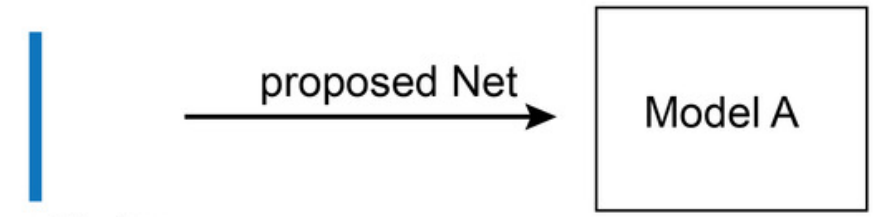

$$
z=64 \text { slices }
$$
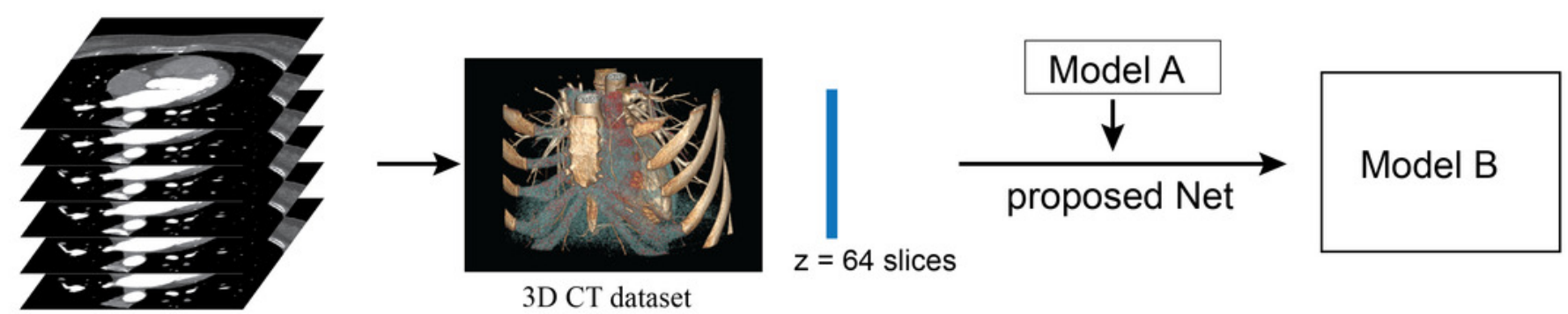

3D CT dataset 


\section{Figure 4}

Fig 4. Example of segmentation result of proposed method on non-contrast CT images.

The first, second, and third rows contain a source dataset in axial view, segmentation result in axial view, and segmentation result in 3D reconstruction, respectively. The yellow color represents the segmented ECF using the proposed method and green color represents the ground-truth. The DSC values for the volume sets from left to right are $90.90 \%, 88.63 \%$, $95.31 \%$ and $87.86 \%$, respectively. The fat volumes from left to right are $95.69 \mathrm{ml}, 116.95 \mathrm{ml}$, $106.99 \mathrm{ml}$ and $91.18 \mathrm{ml}$, respectively.
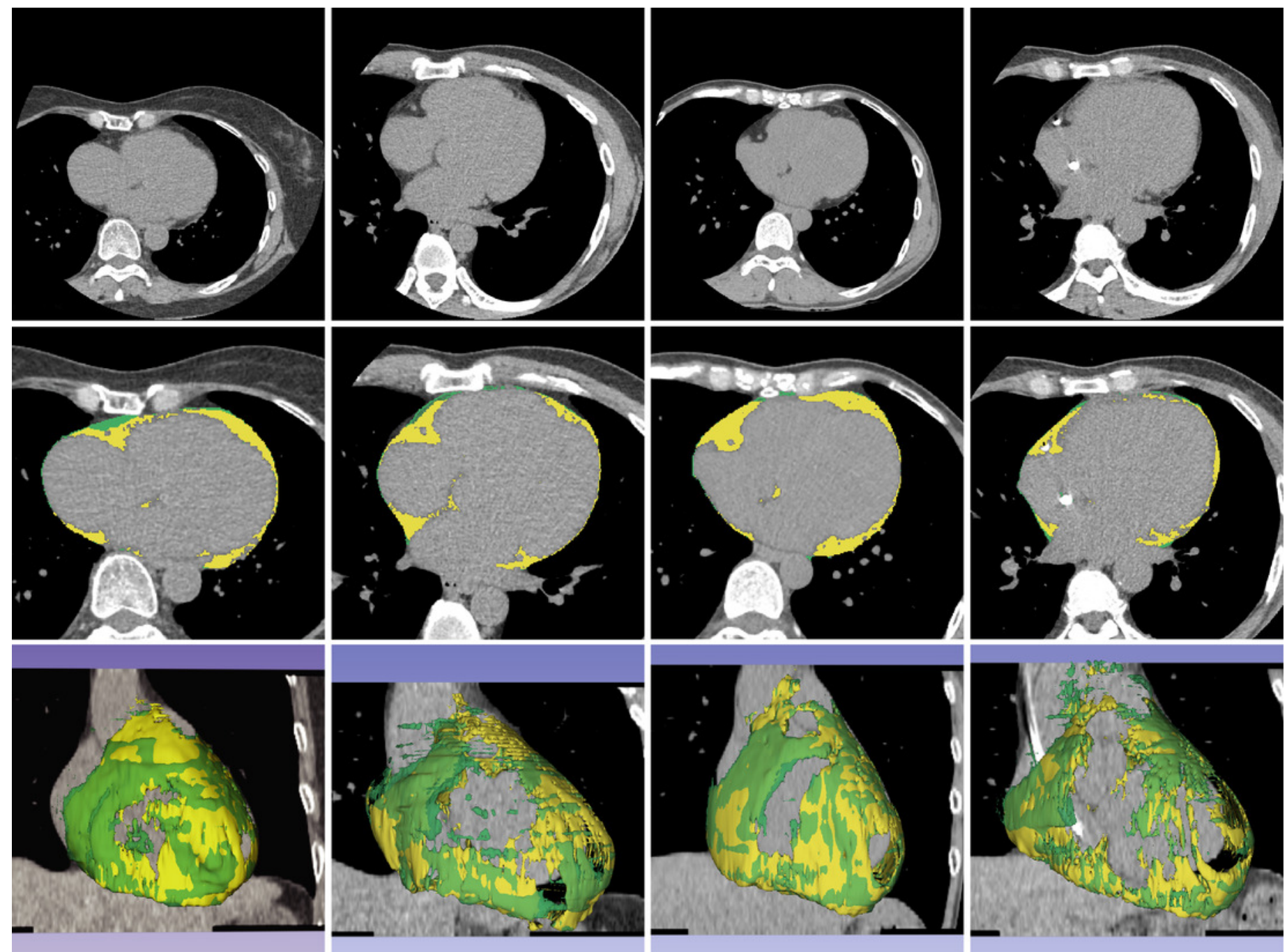


\section{Figure 5}

Fig 5. Example of segmentation result of proposed method on contrast-enhanced CT images.

The first, second, and third rows contain a source dataset in axial view, segmentation results in axial view, and segmentation results in 3D reconstruction. The yellow color represents the segmented ECF and green color represents the ground-truth. The DSC values for the volume sets from left to right volume-sets are $80.23 \%, 93.38 \%, 72.26 \%$ and $92.72 \%$, respectively. The fat volumes from left to right volume-sets are $201.20 \mathrm{ml}, 112.48 \mathrm{ml}, 92.28 \mathrm{ml}$ and $112.98 \mathrm{ml}$, respectively.
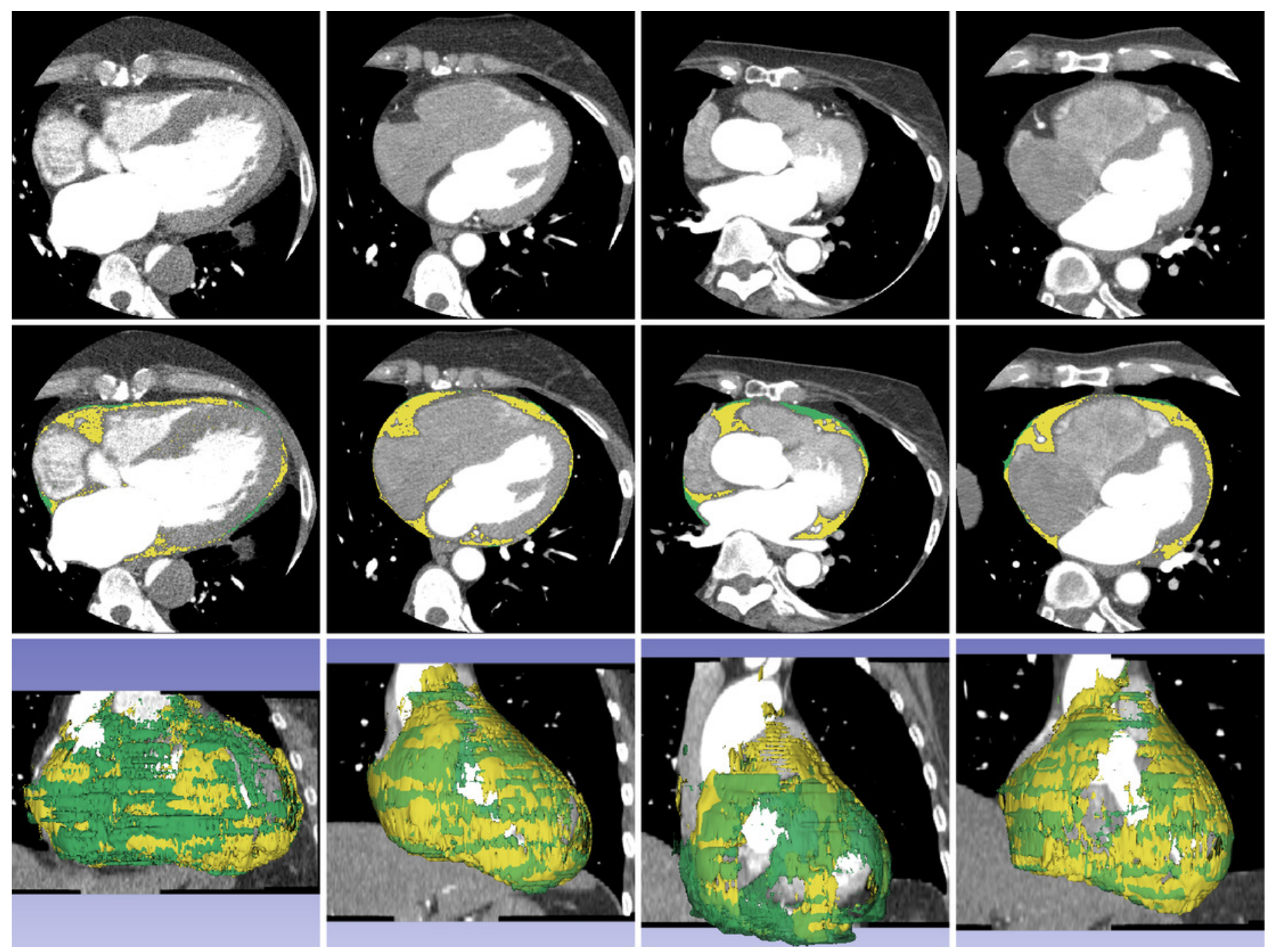


\section{Table $\mathbf{1}$ (on next page)}

Patient characteristics of the CNN non-contrast and contrast-enhanced CT datasets

Patient characteristics of the CNN non-contrast and contrast-enhanced CT datasets 
1 Table1: Patient characteristics of the CNN non-contrast and contrast-enhanced CT datasets

\begin{tabular}{|c|c|c|c|c|}
\hline & \multicolumn{2}{|l|}{ Non-contrast CT } & \multicolumn{2}{|c|}{ Contrast-enhanced CT } \\
\hline & Training dataset & Testing dataset & Training dataset & Testing \\
\hline No. of records & 200 & 20 & 20 & 20 \\
\hline $\begin{array}{l}\text { Average age } \\
\text { (years) }\end{array}$ & $61.41 \pm 12.27$ & $67.80 \pm 11.66$ & $65.85 \pm 8.36$ & $60.75 \pm 10.31$ \\
\hline $\begin{array}{l}\text { Average volume } \\
(\mathrm{ml})\end{array}$ & $135.75 \pm 60.09$ & $127.59 \pm 35.51$ & $117.13 \pm 69.29$ & $121.43 \pm 40.21$ \\
\hline Min volume (ml) & 6.39 & 71.86 & 47.34 & 66.03 \\
\hline Max volume (ml) & 327.44 & 208.20 & 374.82 & 201.20 \\
\hline
\end{tabular}

2

3

4

5

6

7

8

9

10 


\section{Table 2 (on next page)}

Results of 5-fold cross-validation. Mean Dice score coefficient and standard deviation were used to assess model validity and repeatability on the non-contrast CT dataset.

Results of 5-fold cross-validation. Mean Dice score coefficient and standard deviation were used to assess model validity and repeatability on the non-contrast CT dataset. 
2 Table 2. Results of 5-fold cross-validation. Mean Dice score coefficient and standard deviation 3 were used to assess model validity and repeatability on the non-contrast CT dataset.

\begin{tabular}{|l|l|l|l|l|}
\hline Fold & U-Net & AG-U-Net & DSV-U-Net & AG-DSV-U-Net \\
\hline 1 & 87.48 & & & (Proposed method) \\
\hline 2 & 89.97 & 87.93 & 87.70 & 89.55 \\
\hline 3 & 89.22 & 89.65 & 89.93 & 90.73 \\
\hline 4 & 89.50 & 89.76 & 88.30 & 88.76 \\
\hline 5 & 86.44 & 90.08 & 85.56 & 89.61 \\
\hline mean & 88.52 & 84.77 & 82.84 & 86.46 \\
\hline
\end{tabular}

4 


\section{Table 3 (on next page)}

Table 3. Experimental results with standard 3D U-Net, AG-U-Net and the proposed method (AG-DSV-U-Net) on the holdout non-contrast CT dataset

Table 3. Experimental results with standard 3D U-Net, AG-U-Net and the proposed method (AG-DSV-U-Net) on the holdout non-contrast CT dataset 
1 Table 3. Experimental results with standard 3D U-Net, AG-U-Net and the proposed method (AG-

2 DSV-U-Net) on the holdout non-contrast CT dataset

\begin{tabular}{|l|l|l|l|l|}
\hline $\begin{array}{l}\text { Non- } \\
\text { contrast CT }\end{array}$ & U-Net & AG-U-Net & DSV-U-Net & AG-DSV-U-Net \\
\hline DSC & $84.87 \pm 5.73$ & $89.59 \pm 4.45$ & $89.70 \pm 4.81$ & $90.06+4.60$ \\
\hline JSC & $74.12 \pm 8.08$ & $81.41 \pm 6.77$ & $81.64 \pm 7.33$ & $82.21+6.91$ \\
\hline HD & $0.34+0.18$ & $0.27+0.12$ & $0.28+0.14$ & $0.25+0.14$ \\
\hline
\end{tabular}

3

4

5

6

7

8

9

10

11

12

13

14 


\section{Table 4(on next page)}

Table 4. Transferred learning from pre-trained model to contrast-enhanced CT dataset

Table 4. Transferred learning from pre-trained model to contrast-enhanced CT dataset 
1

2 Table 4. Transferred learning from pre-trained model to contrast-enhanced CT dataset

\begin{tabular}{|l|l|l|l|l|}
\hline & U-Net & AG-U-Net & DSV-U-Net & AG-DSV-U-Net \\
& & & & (Proposed method) \\
\hline DSC & $85.58+4.99$ & $82.47+4.33$ & $85.07+4.96$ & $88.16+4.57$ \\
\hline JSC & $75.11+7.19$ & $70.39+6.03$ & $74.32+7.07$ & $79.10+6.75$ \\
\hline HD & $0.41+0.36$ & $0.34+0.23$ & $0.35+0.30$ & $0.28+0.20$ \\
\hline
\end{tabular}

3 